\title{
NACIONALISMO E INTERNACIONALISMO EN LAS CIENCIAS DE LA SALUD: EL CASO DE LA LUCHA ANTIPALÚDICA EN LA ARGENTINA
}

\author{
Alfredo G. Kohn Loncarica \\ Director Depto. de Humanidaddes Médicas y Catedrático de Historia de la Medicina. \\ Facultad de Medicina - Universidad de Buenos Aires. \\ (Av. Salvador M. del Carril 3782, -c. p. 1419- Buenos Aires, Argentina).
}

\author{
Abel L. Agüero \\ Docente Autorizado. Depto. Humanidades Médicas - Facultad de Medicina. \\ Universidad de Buenos Aires \\ Norma Isabel Sánchez \\ Docente Libre y Adscrita. Depto. Humanidades Médicas - Facultad de Medicina. \\ Universidad de Buenos Aires
}

\section{RESUMEN}

El médico sanitaristo Carlos Alberto Alvarado (1904-1986) realizó una obra que dio como resultado la erradicación del paludismo en la Argentina y puede presentarse como una ejemplificación de la espinosa cuestión del nacionalismo y el internacionalismo en la ciencia. Exponen los autores que la experiencia que protagonizó constituye una notable muestra de la inconveniencia de transplantar automáticamente a cualquier área, técnicas y procedimientos científicos eficaces en otros medios, siendo conveniente el desarrollo de investigación propia en los países periféricos.

\section{SUMMARY}

The sanitary physician Carlos Alvarado Albert (1904-1986), accomplished a work that resulted in the eradication of malaria in Argentina. His work could come as an example of the thorny question of nationalism and internationalism in science. The authors of this work expound that the experience that the played the mentioned doctor, constitutes a remarkable sample of the inconvenience of transplanting on an automatic way any technical area and scientific effective procedures to other places, when he in fact what is convenient for the peripheral countries is the development of its own investigation. 


\section{INTRODUCCIÓN}

Hace ya casi una década, la intelectual argentina Hebe Vessuri definía algunas características de la interacción entre la ciencia y la cultura en los países subdesarrollados y, en especial, en Latinoamérica ${ }^{1}$. Según esta autora, una visión puramente economicista de la ciencia produciría una serie de peticiones de principios que resumidamente podrían ser enunciadas del siguiente modo:

1.- La ciencia debe ser concebida como un conocimiento universal de los fenómenos naturales.

2.- En concordancia con lo anterior: al ser las leyes científicas igualmente válidas de la misma manera en toda la geografía del planeta, tornan irrelevantes los contextos culturales en los que se desarrollan.

3.- Por consiguiente, si la cultura es solamente considerada un acompañamiento superestructural de los fenómenos científicos, se encuentra plenamente justificado el obviar estos aspectos y adoptar en forma acrítica las conclusiones de la ciencia y la tecnología de los países centrales.

Los anteriores postulados pueden ser discutidos desde el campo de las llamadas "ciencias duras", pero muestran ser aún más falibles en el terreno de las ciencias sociales, en las cuales es de relevante importancia la cosmovisión que del hombre y su entorno cada cultura ha cimentado a través de su particular evolución diacrónica.

Quedan entonces planteados dos problemas fundamentales a resolver por los científicos de los países marginales: el derecho a la creatividad con una impronta propia y la importación de las ventajas tecnológicas sin que ello conlleve un desastre cultural $^{2}$.

La medicina, disciplina cuyo conjunto de conocimientos oscila entre las fronteras de la ciencia y la técnica y de lo biológico y lo social, ofrece en su desarrollo numerosos ejemplos acerca de lo anteriormente dicho. En esta oportunidad analizaremos un caso que evaluamos como paradigmático: la obra de un visionario periférico, aún en nuestro propio país, pues su acción se desarrolló en las lejanas provincias del noroeste. Nos referimos a Carlos Alberto Alvarado, quien - pese a todas las limitaciones de su ámbito y de su época- pudo resolver importantes problemas sanitarios argentinos utilizando para ello estudios autóctonos y procedimientos que las tradiciones

1 VESSURI, H. (1986), "Los papeles culturales de la ciencia en los países subdesarrollados". En: SALDAÑA, J.J. (Editor). El perfil de la ciencia en América (1986), México, 7-17.

2 Ibidem. 


\section{NACIONALISMO E INTERNACIONALISMO EN LAS CIENCIAS DE LA SALUD}

locales aceptaron por estar íntegramente adaptados al entorno. De las múltiples producciones de este sanitario, nos detendremos en el estudio de su lucha antimalárica.

\section{EL HOMBRE}

Carlos Alberto Alvarado nació el 4 de noviembre de 1904 en la ciudad de San Salvador de Jujuy, en la frontera del altiplano compartido con la República de Bolivia 3 .

Jujuy constituye junto con las provincias de Salta, Tucumán, Santiago del Estero, Catamarca y La Rioja, la región conocida como el Noroeste Argentino (NOA). Avanzada de la colonización durante los tiempos hispánicos, la región fue una zona relativamente próspera durante los años del Virreinato del Río de la Plata, por ser área de enlace entre las ricas tierras del Potosí y Perú, con el puerto de Buenos Aires. Iniciada la época independiente e instituido en la Argentina el modelo económicocultural agroexportador y europeizante, el NOA se transformó en la región más pobre del país, que a los ojos de la capital poseía tres signos indudables de su atraso: el

3 Gran parte de los datos que se exponen fueron tomados de: SIERRA IGLESIAS, J.P. (1987), Carlos Alberto Alvarado, su contribución a la medicina sanitaria. Buenos Aires, tesis de doctorado en medicina, (inédita), 3 tomos (apadrinada por A. G. KOHN LONCARICA). Ha sido publicada como Carlos Alberto Alvarado. Vida y Obra (1994), Salta, C.A.S. (Comisión Bicameral Examinadora de Obras de Autores Salteños), 633 p. Cfr.:

- AlVARADO, C.A. (1947), Resumen biográfico (folleto). San Salvador de Jujuy.

- BoKSTEIN, D. (1953), "Apuntes básicos para la futura historia del Ministerio de Salud Pública". Bs As, Mundo Hospitalario, $\mathrm{N}^{\circ} 114,27-32$.

- HACKETT, L.W. (1945) Alocución en la ceremonia de entrega al Dr. C. A. Alvarado del grado "Honoris Causa" por la Universidad de Portland. Bs As, (folleto mimeografiado).

- HuARQue Falcón, J. "Dr. Carlos Alberto Alvarado. La erradicación del paludismno en el NOA", diario Pregón, (S.S. Jujuy, 22 de agosto de 1988).

- LUNA, F. Perón y su tiempo. Bs As, Compañía Impresora Argentina, To I, p. 404-ss.

- Rodriguez CASTElls, H. (1974), "Discurso de incorporación del miembro honorario Nacional C.A. Alvarado". Bs As, Boletín de la Academia Nacional de Medicina, $\mathbf{N}^{\circ} 52$.

- SiERRA IGLESIAS, J.P. (1985), "Carlos Alberto Alvarado. Apuntes para su biografía” (inédito), VI Congreso de Historia de la Medicina Argentina. San Miguel de Tucumán.

- VERNA, L.C. (1965), "Tres epidemiólogos de Latinoamérica. Osvaldo Cruz, Carlos Chagas y Carlos Alberto Alvarado". Bs As, Boletín de la Asociación Argentina de Microbiología. Año V, № 12. — Pautas (diario S. S. Jujuy): "Jujeño de hoy: Dr. Carlos Alberto Alvarado", marzo de 1979.

- Organización Mundial de la Salud (Press). Dr. Emilio Pampana WHD Malaria Director retires. Dr. Carlos A. Alvarado new director. Ginebra, 26 de octubre de 1964.

- SÁNCHEZ, N. I. (1984), "Pioneros en la lucha contra el paludismo en el norte argentino". En: Sociedad de Historia de las Ciencias de la Salud, Mendoza. Ateneo, 68-80. 
biotipo racial predominante entre su habitantes ${ }^{4}$, la cultura hispano-colonial y el acendrado catolicismo. No era entonces (ni en gran medida lo es aún) el NOA una ambiente propicio para el desarrollo de una mentalidad científica entre sus paisanos.

Alvarado cursó los estudios primarios y secundarios en su ciudad natal, para emprender en 1923 el viaje de casi $1.500 \mathrm{~km}$ al sur con objeto de estudiar medicina en la Universidad de Buenos Aires. Se graduó en 1928 con diploma de honor.

Tras una corta estadía en su provincia, partió becado para especializarse en la Scuola Superiore di Malariologia de Roma, bajo la dirección de los reputados especialistas V. Ascoli, Alessandrini y Missiroli. Por entonces, Italia era uno de los lugares de referencia para perfeccionarse en paludismo, pues en esa época el gobierno fascista había fijado como objetivo la erradicación del mal en la península. Vencidas serias dificultades (la escuela no quería aceptar más latinoamericanos por su conducta disipada), Alvarado terminó sus estudios con la calificación de ottimo con lode en 1929. Tuvo en esos años oportunidad de conocer al Dr. Lewis H. Hackett, quien sería después director regional de la Fundación Rockefeller y uno de sus más entusiastas defensores. Renovada su beca, pasó a la London School of Hygiene and Tropical Medicine, diplomándose en 1930.

A su regreso se instaló en Jujuy y el Departamento Nacional de Higiene lo nombró médico de zona de la lucha antipalúdica (1931). En 1933 reemplazó a su tío, el Dr. Ricardo Alvarado, como director regional de paludismo y jefe de la sección profilaxis de la peste (región norte), comenzando una exitosa acción que será comentada en este trabajo. Basta ahora mencionar que los resultados de su gestión se tradujeron en la erradicación del paludismo en la Argentina, la profilaxis del bocio endémico, del tracoma y de la anquilostomiasis, el plan Koch de lucha antituberculosa, la lucha contra la fiebre amarilla selvática y la vigilancia epidemiológica de la peste, el tifus exantemático y la viruela.

Paralelamente a sus actividades de sanitario, desarrolló una intensa actividad científica. Encontramos su nombre asociado a la creación de la Facultad de Medicina de la Universidad de Tucumán (de la que fue profesor), a la Sociedad Argentina de Patología y Epidemiología de las Enfermedades del Norte Argentino, a la Universidad de Buenos Aires, donde se doctoró en $1941^{5}$ y a la Universidad de Portland, que le concedió el título de doctor honoris causa en 1945. Autor de varios libros y nume-

\footnotetext{
4 Los "cabecitas negras" en el lenguaje de las regiones inmigratorias y de las grandes ciudades litoraleñas, pero en realidad mestizos de blanco e indio, también llamados "criollos" modificando el sentido antiguo de éste término (que definía al blanco nacido en América) y de alguna manera equivalentes a los gauchos de la pampa húmeda.

5 Tituló a su tesis "Tratamiento del paludismo" (Bs As, Imprenta Mercali, 1941) y apareció como libro (Bs As, El Ateneo, 1941), del que hay más de una edición.
} 
rosos artículos, en 1974 recibió el premio "Consagración Nacional”, el mayor galardón académico argentino de carácter oficial.

Contó con el entusiasta apoyo del ministro de Salud Pública Dr. Ramón Carrillo y del propio presidente Juan Perón, pero advirtió que para desarrollar sus planes se interponían un conjunto de circunstancias adversas y renunció a sus cargos, pasando a desempeñar el cargo de Director de la Sección Erradicación de la Malaria de la Organización Mundial de la Salud, hasta su jubilación en 1964. Volvió entonces a Jujuy y por un corto período se alejó de la medicina.

Poco duró su descanso; en 1966 la provincia de Jujuy, a la sazón intervenida, como todas las demás, por el gobierno militar, le convocó para ocupar el Ministerio de Salud Pública. Desarrolló entonces su Plan de Salud Rural, verdadero anticipo de la estrategia de Atención Primaria de la Salud, que doce años más tarde recomendaría la OMS como técnica para alcanzar la meta de "Salud para todos en el año 2000". Iniciado el Plan de Salud Rural, éste fue continuado por el nuevo gobierno jujeño que asumió en enero de 1967. Alvarado, en tanto, pasó a trabajar como ministro de Salud Pública de Salta y puso en marcha también allí, un Plan de Salud Rural, pese a la oposición del ambiente médico de la provincia. En agosto de 1969, Alvarado debió presentar su renuncia por razones de salud y el programa fue interrumpido hasta 1979, en que fue restablecido.

Ya muy enfermo, y alejado de toda actividad, falleció el 28 de diciembre de 1986. Había acumulado, entre otras distinciones, el nombramiento de miembro de las Academias Nacionales de Medicina de Buenos Aires y de Córdoba, los doctorados honoris causa en Portland y Tucumán y la condición de miembro honorario nacional de la Asociación Médica Argentina.

\section{El PAlUdismo EN la ARgENTINA ANTES de la ACCión DE AlVARAdo}

El paludismo o malaria, vastamente conocido en Europa por su acción destructora, también afectaba a las poblaciones autóctonas americanas, con la particularidad que aquí existía el árbol de la quina, cuya corteza fue el primer remedio eficaz contra el flagelo. Habrá que esperar a los finales del siglo pasado para que aparezcan novedades que permitan avanzar en su definición: Charles Louis Alphonse Laverán aisló el agente etiológico de la malaria (1880), al que identificó como un protozoo. Albert F. A. King fue el primero en sostener el papel de los mosquitos en la difusión de la malaria (1881), dato que confirmaría unos pocos años después Ronald Ross, demostrando el papel del anopheles como huesped intermediario. Por esos años varios médicos latinoamericanos realizaban importantes aportes a la infectología: el cubano Carlos Finlay leía ante la Academia de La Habana su comunicación "El mosquito, hipotéticamente considerado como agente de transmisión de la fiebre amarilla" 


\section{ALFREDO G. KOHN LONCARICA, ABEL L. AGÜERO Y NORMA ISABEL SÁNCHEZ}

(1881) y el franco-venezolano Luis D. Beauperthuy, nacido en la antillana isla de Guadalupe, señalaba la relación mosquito/fiebre amarilla ${ }^{6}$. En tanto, en Italia surgían trabajos fundamentales (entre varios, destacaremos el de Grassi y Bastianelli -1883-) que revelaron el ciclo del paludismo en el hombre y la importancia que, en su desenlace, tienen las hembras del mosquito anofélico.

En el caso particular argentino, la historia del paludismo ha pasado por diversas etapas? ${ }^{7}$.

* En un primer momento ("época oscura") se desconocía el ciclo de transmisión de la enfermedad y su profilaxis. La bibliografía se reducía a algunos estudios de la década del ' 80 y fundamentalmente a los de Eliseo Cantón ${ }^{8}$, donde a través de la geografía médica se describían las regiones afectadas y el hallazgo, por parte de Carlos Malbrán, del hematozoario de Laverán en la Argentina.

* La segunda etapa, "época de la toma de conciencia", comienza en torno a 1900, año en que un serio brote palúdico afectó a Santiago del Estero y se prolongó hasta 1903. Por entonces, E. Cantón había viajado a Italia e informaba por correspondencia

6 Otros médicos hispanoamericanos de la época consagraron su vida para estudiar las enfermedades infecciosas: el cubano Juan Guiteras, el colombiano Roberto Franco, son algunos de ellos (Cf: LAíN ENTRALGO, P. (1974), Historia Universal de la Medicina. Barcelona, Salvat, Tomo 6, p. 170).

7 Cf.: - AlvarADO, C. A. Tratamiento del paludismo (Tesis...).

- Tratamiento del paludismo (1974), Bs As, El Ateneo.

- Un programa de erradicación del paludismo en la República Argentina (1947), Tucumán.

- Plan definitivo (1949), Bs As, folleto.

- El justicialismo en el saneamiento urbano y rural (1951), Santa Fe, Universidad Nacional del Litoral (Cátedra Libre de Sanidad Justicialista).

- Alvarado, C.A. y Coll, H. A. (1974), El aspecto asistencial de la lucha antipalúdica, con especial referencia al plan organizado en la República Argentina. Conferencia Sanitaria Panamericana. Cfr. además:

- SONIS, ABRAAM y colaboradores. Medicina sanitaria y administración de salud. Bs As, El Ateneo, 2 tomos. Del T ${ }^{\circ}$ I: Alvarado, C.A., "La erradicación del paludismo"; del To II, Alvarado y TANONI, "Atención de la salud en las áreas rurales y el Plan de Salud Rural de la provincia de Jujuy". 198.

8 El paludismo y su geografia médica en la República Argentina, (1891), Bs As, Imprenta "La Universidad". Obra laureada con el primer premio en el concurso nacional de medicina propiciado por el Círculo Médico Argentino.

E. Cantón se doctoró en Bs As con su tesis "Estudio sobre el paludismo en la provincia de Tucumán", en 1886. Hubo una sola tesis anterior referida a la enfermedad en el país: la de Martín Spuch, "El paludismo en la constitución médica de Buenos Aires" (1875, 56 pág.). Hay otras, pero no referidas exclusivamente al caso argentino (vg. las de Oliva Moisés (1856), Ignacio Ortiz (1877), José M. Juárez (1878), Eudoro Cisneros (1880), Pedro Biscayart (1882). (Cfr.: Univ. Nac. de Bs As, Fac. de Ciencias Médicas. Catálogo de la colección de tesis, 1827-1917 (1918), Bs As, Talleres Gráficos A.Flaiban, 492 p.).Cantón escribió un tercer libro referido al paludismo: "El parásito de las fiebres palustres. Profilaxia y nuevo método para curar el chucho" (1894). 
a sus discípulos sobre las novedades que en la península se producían acerca del paludismo9. En 1901, Félix Garzón Maceda, desde Córdoba, y en 1902 Juan Carlos Delfino, desde Buenos Aires, comenzaron a esclarecer la etiología, propagación y tratamiento de la malaria en nuestro país ${ }^{10}$. A estos nombres habría que agregar, en años posteriores, los de Carlos Vera, Carlos M. Albarracín, Carlos Canavesio, Miguel A. Figueroa, Antonio Filardi, Pedro J. García, Héctor Quintana y Guillermo Ruzzo ${ }^{11}$.

Finalmente, el 31 de julio de 1911 se promulga la Ley $\mathrm{N}^{\circ} 5.195$ de defensa contra el paludismo, que durante treinta años estuvo vigente.

Por esa época, los estudios de entomología médica de Félix Lynch Arribálzaga y Guillermo C. Paterson determinaron que el mosquito vector en la Argentina era el Anopheles pseudopunctipennis ${ }^{12}$.

* Entre 1915 y 1935 el influjo de la escuela malariológica italiana impuso en la Argentina lo que Alvarado llamó "la época de la quinina y del desagüe". La Fundación Rockefeller prestó colaboración, se instalaron las direcciones regionales de paludismo del NOA y se emprendieron grandes obras de ingeniería para desecar pantanos, a imitación de lo efectuado en Europa. Paradójicamente, el paludismo continuó reinando en la región a pesar de los esfuerzos.

En aquel momento, Alvarado se encuentra ya instalado en el norte y reorganizando los servicios (poniendo fin a lo burocrático y dándole más impulso a lo técnicocientífico). A partir de instante, la siguiente etapa queda bajo su mirada abarcativa y controladora.

\section{Alvarado ASUME la lUCha ANTIMALÁRICA}

Cuando Alvarado se incorporó a la lucha antipalúdica, a comienzos de la década de 1930, la situación estaba estancada al no prosperar las técnicas europeas sobre nuestra realidad. Este flagelo no era patrimonio excluyente de nuestro país; en general, en la América Latina era una preocupación recurrente y, sólo para corroborar lo afirmado, recordemos que otros americanos estaban interesados en la misma época por estudios semejantes: nos referimos a Juan I. Jiménes Grullón, de Santo Domingo,

9 SIERRA IGLESIAS, J. P._Obra citada, tesis, T ${ }^{\circ}$ I, p. 19

10 Ibidem.

11 Ibidem.

12 PAterson, G. C. (1911), "Las fiebres palúdicas de Jujuy". Bs As, Anales del Departamento Nacional de Higiene, Vol. XVIII, 1911, 31-56. El Dr. Paterson, era hacia 1894 un joven médico inglés que se trasladó al ingenio La Esperanza (Jujuy) y allí continuó la labor inconclusa de Cantón y tuvo el mérito de ser el identificador de los tres plasmodios. (Cf:: SIERRA IGLESIAS, J. P. (1978), Vida y obra del Dr. Guillermo C. Paterson (Padre de la Patología Regional Argentina). Tucumán, Univ. Nac. de Tucumán, Fac. de Medicina, 185 p.). 
quien en 1929, estando en París, presentó su tesis Plan d' organisation de la lutte antipaludéenne $e^{13}$ o al Dr. Moscoso Carrasco, del Servicio de Paludismo, que desde la ciudad de Cochabamba, dirigió la lucha antipalúdica en Bolivia ${ }^{14}$.

Un somero análisis de la geografía médica argentina mostraba que en el territorio existían dos áreas maláricas de diferentes características ${ }^{15}$. El paludismo era endémico en el NOA, región andina y pre-andina que comprendía unos $120.000 \mathrm{~km} 2$, con una población de 850.000 habitantes, de los cuales el $65 \%$ eran pobladores rurales. El 50\% de los infectados se concentraban en la provincia de Tucumán y en las restantes había zonas palúdicas y zonas indemnes. La acción del Departamento Nacional de Higiene llegaba al $1 \%(1.200 \mathrm{Km} 2)$ del total del área afectada.

En el noreste argentino (NEA) se encontraba la franja del paludismo epidémico con pequeños focos endémicos, que afectaba las provincias de Misiones, Corrientes, Chaco, Formosa y Santa Fe, con una extensión también de 120.000 km2.

La lucha antipalúdica había sido dirigida desde Buenos Aires por el Departamento Nacional de Higiene y según el modelo italiano de los bonifica (saneamiento). Cuenta el mismo Alvarado que, durante sus permanencia en Italia, oyó a Mussolini referirse a la desecación de las Paludes Pontinas en estos términos:

"Bonificare la terra per bonificare l'uomo, bonificare l'uomo per bonificare la razza"16.

A su vez, los bonifica se calificaban según su acción en:

a) Bonifica hidráulica: solución de los ingenieros, basada en canales de desecación de pantanos, que a su vez se convirtieron en criaderos de mosquitos exigiendo

\footnotetext{
13 Hemos seleccionado el nombre de Jiménes Grullón (médico con auténticos intereses por la filosofía y cuya labor cubrió tanto a Santo Domingo como a Cuba, patria esta última que adoptó por las persecuciones políticas de Trujillo) porque sabemos del compromiso profesional y político que asumió. ( $C f$. Universidad Autónoma de Santo Domingo. Fundación Federico C. Alvarez. La utopía de América (homenaje a Leopoldo Zea -Memorias-). Santo Domingo, Simposio Internacional sobre el Quinto Centenario, 253 p. Ver trabajo de Miguel Rojas, "Significación y permanencia de la filosofía de la libertad de Juan I. Jiménes-Grullón", 213-233).

14 En el $\mathrm{III}^{\circ}$ Congreso de Historia de la Medicina, realizado en Tarija (Bolivia), 2-4 diciembre de 1994, hubo una mesa redonda, presidida por el Dr. César Moscoso (de Cochabamba), con el tema "Historia de la Malaria". Se hizo allí mención a la labor de Moscoso Carrasco. Estas ponencias están inéditas, pero en vías de publicación.

15 Alvarado, C.A. (1944), "Geografía médica del paludismo en la República Argentina". Bs As, Sociedad Argentina de Estudios Geográficos (GAEA), № 9, 1-12.

16 Mencionado por SieRra IGLESIAS, Obra citada (tesis), T ${ }^{\circ}$. I, p. 52.
} 
la instalación de bombas y el espolvoreo con verde de París o petróleo para destruir las larvas;

b) Bonifica humana: propuesta de los médicos, que intentaron eliminar el parásito de la sangre mediante el uso extensivo de la quinina, previniendo la infección en los mosquitos. Al no matar a los esporozoitos descendía la morbilidad pero no la mortalidad;

c) Piccola bonifica: combinaba la administración de quinina con los drenajes de aguas y el uso de larvicidas. Fue el más empleado en la Argentina, pero fracasó por no adaptarse la técnica a la biología del mosquito autóctono, ni al ambiente geográfico y humano.

d) Bonifica integral: propiciaba colonizar las tierras palúdicas para sanearlas con fines económicos. Presuponía necesariamente el cultivo intensivo de la tierra, lo cual era inaplicable en una zona tan vasta como la nuestra.

El fracaso de la prevención, patentizado con la epidemia de Villa Monteros de 1933, donde hacía 10 años que se efectuaba la piccola bonifica, llevó a Alvarado —ahora director de la Dirección Regional de Paludismo de Jujuy - a convencerse de que eran necesarios estudios nacionales para enfrentar la malaria. Comenzó, entonces, a volcar su atención sobre el mosquito.

De todos los anofelinos del NOA, estaba ya bien establecido que el vector palúdico era solamente el Anopheles pseudopunctipennis. Este mosquito es doméstico, prefiere atacar al hombre y convive con él en cualquier tipo de habitación. A diferencia del resistente adulto, las larvas son muy lábiles. Alvarado inició los estudios por el vector y no por el plasmodio.

Organizó, de inmediato, un equipo de trabajo, contratando peones de la zonas ${ }^{17}$. En 1932, se tenía bajo observación una zona periurbana de San Salvador de Jujuy, cubriendo a 16.000 habitantes. Este radio fue dividido en secciones, cada una a cargo de un peón con capacidad de recorrer todos los charcos de agua en una semana y destruir las larvas de A. pseudopunctipennis, antes de que se convirtieran en ninfas (ciclo que requiere 10 días). Esta acción se controlaba instalando estaciones de captura de anófeles para comprobar si descendía su número.

17 Alvarado, C. A. (1932), Sobre el índice palúdico de la ciudad de Jujuy y alrededores, levantado en abril de 1932 y otras observaciones sobre el problema palúdico realizadas dentro de la última época epidémica (julio 1930-junio 1932). Informe a máquina, Jujuy, agosto.

- Informe sobre los resultados de la última campaña antipalúdica en la ciudad de Jujuy (1934. 1935) elevado a la presidencia del Departamento Nacional de Higiene. Jujuy, 1935.

- Alvarado, C. A. y Susini, M. (1935), "El paludismo en la Argentina. Contribución al conocimiento de la biología del Anopheles pseudopunctipennis". Buenos Aires, Anales del Departamento Nacional de Higiene, Vol. XXXVI, 5-20. 
Para sorpresa de Alvarado, las larvas recogidas eran casi todas de una especie inofensiva, el $A$. argyritarsis, aunque las estaciones de captura mostraban un alto índice de pseudopunctipennis. Extendiendo el espacio de inspección, se pudo encontrar por fin el esquivo criadero de mosquitos vectores: las playas de los arroyos y un espléndido canal de riego con paredes de piedra. Profundizando las observaciones llegó a la conclusión, ya entrevista por Paterson en $1911^{18}$, de que los criaderos de $A$. pseudopunctipennis se hallaban en los cursos de aguas rápidas, libres de vegetación y con taludes verticales y limpios, que se encontraban en tan buena situación como efecto de los trabajos de bonifica anteriores. Aquellas vertientes que, por el contrario, no habían sido desmalezadas solo producían $A$. argyritarsis.

Tras varios ensayos de hipótesis, Alvarado postuló que para el desarrollo de sus larvas, el A. pseudopunctipennis necesita ausencia de vegetación acuática vertical, aguas bien aireadas y soleadas en constante renovación y la presencia del alga spirogirae que le da alimento y protección. Circunstancias que, irónicamente, se facilitaban con la bonifica hidráulica o la piccola bonifica.

La solución propuesta consistió en "renaturalizar" los cursos de agua plantando berros y lampazos en el lecho y arbustos para sombra en los bordes. El resultado fue la desaparición de las larvas del temido pseudopunctipennis.

La contraprueba no tardó en presentarse. Oigámosla en las propias palabras de Alvarado:

\begin{abstract}
"Un vecino de villa Gorriti... situada sobre las márgenes del río Chico..., se presentó un día protestando por la inercia de la lucha antipalúdica. Expresaba que en los años anteriores le habían desyuyado los canales de la vecindad de su domicilio, que este año no se había hecho todavía, por esa causa todo estaba lleno de mosquitos. Al no convencerse con las explicaciones que se le dieron... amenazó con quejarse a los diarios y hacer él el trabajo. No se sabe si llevó las quejas a los periódicos, pero sí que limpió un trecho de un canal próximo a su casa. Allí, sólo allí, fuera de la playa, se encontraron larvas de App"'19.
\end{abstract}

\title{
5. LA LUCHA ANTIPALÚDICA SE EXTIENDE A TODO EL NOA
}

Fundada en estos nuevos conceptos, la lucha antipalúdica dio rápidos resultados. En 1934 el plan abarcó toda la ciudad de Jujuy y unos años después se completó con la destrucción invernal de larvas por medio del petróleo o del verde de París.

El éxito del llamado "plan Alvarado" se afianzaba, pero para su responsable aumentaban las exigencias: de 1935 a 1937 desempeñó en Buenos Aires la secretaría

18 PATERSON, G. C. Obra citada.

19 Mencionado por SIERRA IGLESIAS. Obra citada (tesis), T ${ }^{\circ}$. I, p. 68. 
del Departamento Nacional de Higiene y más tarde se instaló en Tucumán como director de la Dirección General de Paludismo, creada por ley del Congreso.

Desde su puesto en Tucumán, quedó encargado de la lucha antipalúdica para todo el país. Su metodología de trabajo ${ }^{20}$ se reducía a tres aspectos fundamentales:

a) El programa de la policía de focos, destinado a eliminar los criaderos anofelinos en un radio de $4 \mathrm{~km}$ alrededor de la zona protegida. Lo que significa que, como el anofeles vuela no más de 4.000 metros, no habría en el radio de protección, ni producción endógena, ni mosquitos que llegaran del exterior.

b) La profilaxis invernal, que se realizaba con la llegada del frío y para esterilizar un radio cada vez más extenso, de forma que al arribar el verano no hubiese posibilidad de reproducción de $A$. pseudopunctipennis.

c) El reparto de quinina en las zonas no protegidas, con la premisa de llegar a todas las familias sin excepción y por más apartados que fueran los lugares de su vivienda.

El control de los resultados, medido por diversos métodos (índice hematológico, índice esplénico, índice anofélico) mostró la eficacia del programa. En pocos años, la Dirección de Paludismo estudió la extensión y precisó los caracteres regionales de la endemia, creó los métodos más eficaces y económicos para combatirla, estudió nuevas drogas antimaláricas, construyó sifones de agua antilarvarios, sembró peces larvófagos y murciélagos para la lucha antianofelina, estableció el papel de los arrozales en la propagación anofelina y fundamentalmente realizó una labor de educación para la salud utilizando canales de comunicación fácilmente entendibles por los habitantes de la zona ${ }^{21}$.

20 Alvarado, C. A. (1938), "Malaria Control in the Argentine Republic". Amsterdam, Actas del III Congreso Internacional de Malaria, Vol. I, 250-252.

- Pautas para la lucha antipalúdica en el país (1938), Boletín de Obras Sanitarias de la Nación. Tomo II, 1938, p. 217.

- Métodos de lucha antipalúdica en la República Argentina. Informe presentado por el Presidente del Departamento Nacional de Higiene, Dr. M. Susini. Boletín Sanitario. Departamento Nacional de Higiene, $N^{\circ}$ III, 1939, 891-904.

- Estado actual de la lucha antipalúdica en el Norte Argentino. Bs As, Folleto del Congreso de Medicina Social y Gremial, julio de 1942.

21 Alvarado, C. A. y DEL PONTE, E. (1938), Sobre la existencia del Anopheles annulipalpis en la Provincia de Mendoza (R.A.). Descripción de su larva. Bs As, Folia Biológica.

- Alvarado, C.A. (1938), Biology of the Anopheles pseudopunctipennis in the Argentine Republic, and methods of control. Amsterdam, Actas del III $^{\circ}$ Congreso Internacional de Malaria, Vol. III, set.oct. 100-102. 
Mientras se desarrollaba la lucha antimalárica con éxito, se originaban en el país situaciones que iban a tener su incidencia sobre el tema. Una de ellas fue política: el advenimiento del peronismo; y la otra fue de carácter técnico: la difusión del insecticida DDT.

En 1943 una nueva revolución había instaurado en la Argentina a otro gobierno militar y, entre sus figuras, comenzaba a destacarse la del coronel J. D. Perón. Ante el peligro que para los cogobernantes militares representaba la popularidad de este último, se intentó desplazarlo del poder y el resultado fue la rebelión popular del 17 de octubre de 1945. Perón volvió al centro de la escena política y al año siguiente fue consagrado electoralmente presidente de la Nación.

Las políticas populistas del peronismo, devenido en gobierno, tuvieron algunos centros de interés prioritarios, entre los que se destacaba la salud. El 23 de mayo de 1946, un acuerdo general de ministros, creó la Secretaría de Salud Pública, al frente de la cual se nombró a quien sería recordado como el gran sanitario del peronismo: el neurocirujano santiagueño Ramón Carrillo ${ }^{22}$. Pocos meses después, Alvarado recibe la petición de realizar un plan quinquenal antipalúdico (1947-1952) que se acoplaría a los planes quinquenales de gobierno del peronismo. La respuesta fue el "Plan 46", publicado en 1947 en el Tomo II del Plan Analítico de Salud Pública ${ }^{23}$.

Este Plan 46 comprendía un programa asistencial, asentado en dispensarios antipalúdicos, servicios hospitalarios y sanatorios/colonias, y un plan preventivo basado en la labor que se estaba realizando. Había entonces que decidir si se apoyaban estas ideas que, a pesar de su éxito en el terreno, todavía despertaban suspiciacias por contradecir a las escuelas europeas.

- Alvarado, C. A. y SilvetTI PeÑa, L. (1944), Aplicación de sifones antilarvarios en la República Argentina. Boletín de la Oficina Sanitaria Panamericana. Año 23, $\mathrm{N}^{\circ} 10$, octubre, 876-886.

- Alvarado, C.A. y DEL PONTE, E. (1945), Investigación sobre la presunta existencia del Anopheles gambiae en el Paraguay. Bs As, Revista del Instituto Bacteriológico Malbrán, T. XIII, 259-266.

- AlvarAdo, C. A. y HeREDIA, R. L. (1947), Observaciones sobre una nueva variedad del Anopheles (A.) pseudopunctipennis-Theobald, 1901, encontrada en la Provincia de Tucumán, República Argentina. (Comunicación previa). Tucumán, Anales del Instituto de Medicina Regional de la Universidad Nacional de Tucumán, Vol. II, $\mathrm{N}^{\circ} 1$, noviembre 73-78.

- Alvarado, C. A. (1941), Arrozales y paludismo. Bs As, La Prensa Médica Argentina, 3 de octubre.

- Alvarado, C. A. (1943), Informe sobre la influencia del cultivo del arroz en el desarrollo del paludismo (folleto), Tucumán.

- Alvarado, C. A. Tratamiento....

22 Alzugaray, R. A. (1988), Ramón Carrillo, el fundador del sanitarismo nacional. Bs As, C.E.A.L., tomo I, 65-ss.

23 Alvarado, C. A. (1946), Plan de lucha antipalúdica quincenal, Tucumán (folleto), 20 de noviembre. 


\title{
NACIONALISMO E INTERNACIONALISMO EN LAS CIENCIAS DE LA SALUD
}

El gobierno tenía dudas y así leemos:

\begin{abstract}
"Carrillo llevó a Alvarado ante el Presidente Perón, para explicarle en detalle el problema, porque se trataba de una cuestión delicada que implicaba un riesgo político, en la medida en que había que enfrentar a los técnicos ortodoxos (y contradecir a los especialistas internacionales), haciendo un giro revolucionario en la programación de la campaña. Un fracaso podría haber significado la muerte política para Carrillo y un golpe para el gobierno justicialista que llevaba pocos meses en el poder"24.
\end{abstract}

El ministro asumió el riesgo de apoyar a Alvarado e incorporó la lucha antipalúdica al programa denominado "Grandes Luchas Sanitarias". Mientras esto ocurría, Alvarado y su equipo de colaboradores comenzaron a experimentar con un nuevo producto larvicida y adulticida: el DDT (1946). Sus resultados fueron tan satisfactorios que en 1947 la División Paludismo y Enfermedades Tropicales, que era el nuevo nombre del ente que dirigía Alvarado, decidió retirar el "Plan 46" para concebir otro basado en una dedetización de dos años ${ }^{25}$.

La puesta en marcha del nuevo plan no fue sencilla: los clásicos peones de la lucha antipalúdica debieron aprender a conducir camiones y a manejar máquinas fumigadoras; en el terreno de la educación popular hubo que inventar una nueva figura de historieta, ella fue el "sargento DDT".

Fue necesario, además, vencer los problemas de logística que el aprovisionamiento de vehículos y máquinas demandaba. No obstante todas las dificultades, el 28 de mayo de 1947 desfilaban en acto público por las calles de San Miguel de Tucumán los camiones, camionetas y acoplados con que se se iniciaría la campaña. Posteriormente llegaron los vehículos livianos de doble tracción, necesarios para penetrar en el monte.

La dedetización en todas las zonas palúdicas comenzó en setiembre de 1947 y se prolongó a lo largo de 1948 y 1949. En este último año se reformó la vieja ley anti-

24 Alzugaray, R. A. Obra citada, To . II, p. 125.

25 AlvarAdo, C. A. (1947), Un programa de erradicación del paludismo en la República Argentina Tucumán (folleto).

- Plan quinquenal de lucha antipalúdica. Dedetización. Funciones y obligaciones de la sección Ingeniería Sanitaria. Ingenieros Regionales, Jefes de sector y Jefes de Brigada (1947), Tucumán (folleto).

- Conceptos sobre el nuevo plan de lucha antipalúdica de la Secretaría de Salud Pública de la Nación (1947-48), Tucumán, publicación 514, (Anuario) de la Universidad Nacional de Tucumán.

- Alvarado, C. A. y COll, H. A. (1949), “Organización y resultados de la campaña antipalúdica que realiza la Dirección de Paludismo y Enfermedades Tropicales del Ministerio de Salud Pública de la Nación". Tucumán, Salta y Jujuy. Actas de la Primera Reunión Panamericana de la Enfermedad de Chagas y Primera Reunión Conjunta de Enfermedades Transmisibles. Tercer Congreso de Enfermedades Transmisibles. 
palúdica de $1905^{26}$. Fue 1949 el año final de la campaña y los resultados estaban a la vista: en 1941 se habían registrado en el país 10.000 casos de paludismo en enero; en el mismo mes de 1949 sólo 202. La Argentina se transformaba en el primer país del mundo que podía prácticamente erradicar el paludismo endémico en su territorio.

En aquel año se disolvió la Dirección General de Paludismo por haber (como decía el decreto $\mathrm{N}^{\circ} 16.700$ ) "cumplido con los fines de su creación o sea la erradicación del paludismo como enfermedad endémica y social del norte argentino".

En su reemplazo se organizó la Dirección General de Sanidad del Norte.

\section{COMENTARIOS Y CONCLUSIONES}

Decíamos al principio de este artículo que los grandes desafíos de la ciencia latinoamericana podían enunciarse como:

a) creatividad con impronta propia;

b) importación de tecnología de avanzada sin que ello afecte la cultura autóctona.

Pasemos, entonces, basándonos en lo expuesto, a analizar la labor de Alvarado en ambos terrenos.

Uno de los grandes méritos del exitoso caso de la lucha antipalúdica en la Argentina se debe al espíritu libre de su ejecutor, que no se sintió "dependiente" de los conceptos de la escuela italiana de malariología, donde él mismo se había formado. Dependencia que en un país como la Argentina tiene orígenes complejos. Uno de ellos se debe a su propia composición demográfica. El fenómeno inmigratorio, por ejemplo, hizo pasar la población de un total de 1.700 .000 habitantes en 1869 a 7.800.000 en $1914^{27}$. El peso del cambio demográfico tiene, como puede estimarse, una intensa significación en la conformación de la sociedad argentina actual. En la época de Alvarado, cuando todavía vivían los extranjeros emigrados y sus primeros descendientes, el mito del eterno retorno que muchos de ellos alentaban, producía una idealización de sus patrias de origen, a las que veían como la culminación perfecta de la ciencia y la cultura. Y entre estas nuevas madres patrias, la italiana figuraba en un lugar preminente. A ello había que sumar la propaganda fascista glorificadora, propia de los años que estamos tratando.

26 Alvarado, C. A. (1942) "Comentarios a los proyectos de nueva ley de profilaxis y lucha antipalúdica”. Bs As, La Semana Médica, № 28, Vol. 49, 76-83.

27 ARMuS, D. (1983), Manual del Emigrante Italiano. Bs As, C.E.A.L., p. 8. 
A pesar de todas las dificultades y de la situación periférica de su campo de acción, Alvarado reclamó y obtuvo el derecho de efectuar estudios independientes y en terreno propio. Pudo así adaptar sus conclusiones al NOA (sin olvidar que tenemos grandes diferencias con Italia como la vastedad del terreno y la escasez de población) y encontrar las soluciones convenientes a la idiosincrasia de sus pobladores.

Particularmente importante resultó en la estrategia la colaboración popular, lo que indica que se emplearon los canales de comunicación adecuados. No se hablaba, por ejemplo, solamente de "paludismo" - palabra extraña en nuestras poblaciones andinas - sino que se empleaba el vocablo quechua chucho, que todos entendían. Otro acierto fue el uso de recursos comunes de la vida diaria. En las décadas de 1930 y 1940 un elemento de decoración en las paredes de las casas de los pobres lo constituía el "almanaque", que además de cumplir su función de marcar los días ilustraba con sus láminas mensuales. Su valor ya había sido comprendido por algunas firmas comerciales como la Fábrica Argentina de Alpargatas que distribuía los suyos con dibujos camperos del pintor Molina Campos. En 1939, Alvarado y sus colaboradores comenzaron a distribuir el "Almanaque Sanitario", con instrucciones sencillas y claras respecto al paludismo y otras endemias. Esta acción se completó con el "juego del mosquito" y la enseñanza en las escuelas para los niños o la instalación de médicos educadores sanitarios.

Convencida la población, ella se transformó en un formidable agente de salud, máxime cuando los métodos de prevención eran los habituales a su estilo de vida.

Sin embargo, las necesidades tecnológicas impusieron un nuevo desafío. El uso del DDT implicaba que los peones rurales debían conducir automotores y manejar máquinas. Consideramos que la nueva campaña tuvo varios puntos cruciales que permitieron su inserción, sin saber a ciencia cierta si sus conductores fueron conscientes de ello. Debe haber impactado fieramente en la población el desfile de camiones que en 1947 entró a la ciudad para convocar a sus hijos a una nueva lucha, pero esta vez contra el mosquito.

Párrafo aparte merece el "sargento DDT", creado por un médico rosarino y caricaturista amateur, el Dr. Moisés Aizemberg, y que se imprimió en láminas, carteles y tarjetas postales con estrofas tan sencillas como la que dirige el sargento a un mosquito contra al que juega al futbol:

\author{
"Ni tus actuales temblores \\ ni tus antiguas bravatas \\ evitarán que el sargento \\ te haga gol entre las patas".
}

La particularidad de "DDT" es que no era ni comisario ni juez de paz, personajes estos "leidos" que vienen de la ciudad para ejercer la autoridad; por el contrario es 
un simple sargento, grado que cualquier paisano metido a "milico" puede alcanzar y que lo identifica con el pueblo.

Alvarado contó, como ya dijimos, en cierta etapa, con el apoyo del peronismo. Indudablemente esta corriente política, y sobre todo el peronismo temprano, tuvo una inocultable simpatía por las derechas autoritarias europeas, algunos de cuyos programas intentó también desarrollar. Al mismo tiempo los objetivos gubernamentales de mejorar la calidad de vida de las masas populares coincidían con los intereses antimaláricos y la posibilidad de realizarlos sobre la base de soluciones propias debió tentar a un gobierno con ideas nacionalistas. La conjunción de factores y la adhesión de Alvarado al justicialismo favorecieron grandemente al desarrollo de la lucha antimalárica, de la cuál un autor distante ideológicamente, como Felix Luna, llegó a decir: "con poco más, un gobierno entero hubiera justificado su paso por el poder" 28 .

Alvarado - a nuestro entender - es una figura fundamental de "sanitarismo" argentina del siglo XX, movimiento (del que puede ser considerado uno de sus fundadores) que ha sido el continuador, con variantes, del "higienismo" del siglo XIX, conformado por un grupo de avanzada y entre los que se encuentran hombres prominentes de la medicina argentina y latinoamericana ${ }^{29}$.

$\mathrm{Al}$ mismo tiempo queda claro el papel que debe jugar el Estado como garante de la salud y la ciencia. Y podríamos parafrasear al lider indio Pandit J. Nehru: "para un país subdesarrollado la investigación no es un lujo sino una necesidad vital".

La malaria —enfermedad que afecta a los países tropicales y subtropicales - se sigue tratando a título preventivo y en estado clínico con quinina y sus sales y derivados sintéticos, y por eso hoy aparece como una esperanza el descubrimiento de una vacuna sintética antipalúdica preparada por el científico colombiano Manuel Elkin Patarroyo ${ }^{30}$.

Concluimos señalando que el caso de la lucha antimalárica en la Argentina constituye un expresivo ejemplo de la necesidad de introducir una adecuada dosis de nacionalismo en la actividad científica. Admitiendo el indudable carácter internacionalista de la investigación científica y de sus aplicaciones, sin embargo es imprescindible: a) desarrollar investigaciones propias y b) realizar adaptaciones nacionales de los adelantos provenientes del exterior evitando transferencias automáticas, con frecuencia destinadas al fracaso.

\footnotetext{
28 LUNA, F. Obra citada. To . I, p. 404.

29 Entre esos nombres pueden ubicarse, en apretada síntesis, Francisco J. Muñiz, Guillermo Rawson, Eduardo Wilde, Emilio Coni, Pedro Mallo, Julio Méndez, José Penna, José M. Ramos Mexía, Pedro N. Arata, Gregorio Araoz Alfaro.

30 En 1994, recibió el premio "Fundación Principe de Asturias", de Investigación científica y técnica. Cf:: Romero, F. (1994), Manuel Elkin Patarroyo (un nuevo continente de la ciencia). Colombia, Tercer Mundo Editores.
} 
El análisis minucioso de la obra de Alvarado puede servir como un elemento más, inspirador de una sana política científica y tecnológica que tienda a preservar y promover nuestros centros de investigación. Las ciencias y técnicas de la salud no deben alejarse de la ciencia y la tecnología en general y no deben confiar en la solución a través de la simple recepción y aprovechamiento de los avances generados en los países que son líderes en el campo de la investigación. 